\title{
The anti-influenza virus drug, arbidol is an efficient inhibitor of SARS-CoV-2 in vitro
}

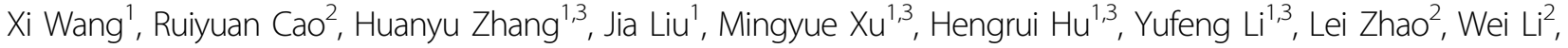 \\ Xiulian Sun ${ }^{1}$, Xinglou Yang ${ }^{1}$, Zhengli Shi', Fei Deng ${ }^{1,4}$, Zhihong Hu (1)', Wu Zhong ${ }^{2}$ and Manli Wang (1)
}

Dear editor,

Since December 2019, a novel disease COVID-19 caused by severe acute respiratory syndrome coronavirus 2 (SARS-CoV-2) rapidly spread to over 200 countries and infected over 1.50 million people including 92,798 deaths (data as of April 10, 2020). On March 11, the World Health Organization (WHO) characterized COVID-19 as a pandemic, and called for accelerating diagnostics, vaccines, and drugs developments to combat this novel disease. Apart of the new coronavirus, influenza virus infections have been a consistent threat to the global public health over the years. In the United States alone, the Centers for Disease Control and Prevention (CDC) estimates that, so far during the 2019-2020 winter season, there have been at least 39 million illnesses, 400,000 hospitalizations and 24,000 deaths from influenza (https://www.cdc.gov/flu/weekly/index.htm). Considering the current concomitant circulation of SARS-CoV-2 and influenza virus infections, the exploration of available and viable anti-influenza drugs to treat both diseases is of great interest.

Actually, in the early stages of the outbreak of COVID19, some anti-flu drugs (for example, oseltamivir) have been applied for the treatment of COVID-19 patients ${ }^{1,2}$. Previously, we reported that favipiravir (T705), an antiinfluenza drug approved in Japan and China, showed a certain efficacy against SARS-CoV-2 in vitro ${ }^{3}$. In addition, arbidol, an anti-influenza drug targeting the viral hemagglutinin (HA) is being used in a clinical trial against

\footnotetext{
Correspondence: Zhihong Hu (huzh@wh.iov.cn) or Wu Zhong (zhongwu@bmi. ac.cn) or Manli Wang (wangml@wh.iov.cn)

${ }^{1}$ State Key Laboratory of Virology, Wuhan Institute of Virology, Center for

Biosafety Mega-Science, Chinese Academy of Sciences, Wuhan 430071, China

${ }^{2}$ National Engineering Research Center for the Emergency Drug, Beijing

Institute of Pharmacology and Toxicology, Beijing 100850, China

Full list of author information is available at the end of the article

These authors contributed equally: Xi Wang, Ruiyuan Cao, Huanyu Zhang
}

COVID-19 (ChiCTR2000029573) and has been recently added to the Guidelines for the Diagnosis and Treatment of COVID-19 (sixth and seventh editions) in China. A recent retrospective study suggested that arbidol treatment showed tendency to improve the discharging rate and decrease the mortality rate of COVID-19 patients ${ }^{4}$. However, to our knowledge, there has been no systematical analysis about the efficacy of anti-influenza drugs against SARS-CoV-2.

In this study, we evaluated six currently available and licensed anti-influenza drugs against SARS-CoV-2. The drugs include arbidol, baloxavir, laninamivir, oseltamivir, peramivir, and zanamivir ${ }^{5,6}$. The M2 inhibitors (amantadine and rimantadine) were not considered in this study since they were not recommended for treating influenza by WHO due to drug resistance. First, the cytotoxicity of the compounds in African green monkey kidney cells, Vero E6 (ATCC-1586) was measured by a standard cell counting kit-8 (CCK8) assay. Then, the cells were infected with SARS-CoV-2 at a multiplicity of infection (MOI) of 0.05 in the presence of either compound or dimethyl sulfoxide (DMSO) control. The dose-response curves were determined by quantification of viral RNA copy numbers in the supernatant of infected cell at $48 \mathrm{~h}$ post infection (p.i.). As demonstrated in Fig. 1a, arbidol efficiently inhibited virus infection in vitro. The $50 \%$ maximal effective concentration $\left(\mathrm{EC}_{50}\right)$ and the $50 \%$ cytotoxic concentration $\left(\mathrm{CC}_{50}\right)$ of arbidol was 4.11 (3.55-4.73) and $31.79(29.89-33.81) \mu \mathrm{M}$, respectively, and the selectivity index $\left(\mathrm{SI}=\mathrm{CC}_{50} / \mathrm{EC}_{50}\right)$ was 7.73 . Baloxavir partially inhibited SARS-CoV-2 infection ( 29\%) at a high concentration of $50 \mu \mathrm{M}$ (Fig. 1a). In contrast, laninamivir, oseltamivir, peramivir, and zanamivir did not exhibit antiSARS-CoV-2 activity even at the highest drug concentrations (Fig. 1a). The antiviral effect of the compounds was also evaluated by observing cytopathic effects 

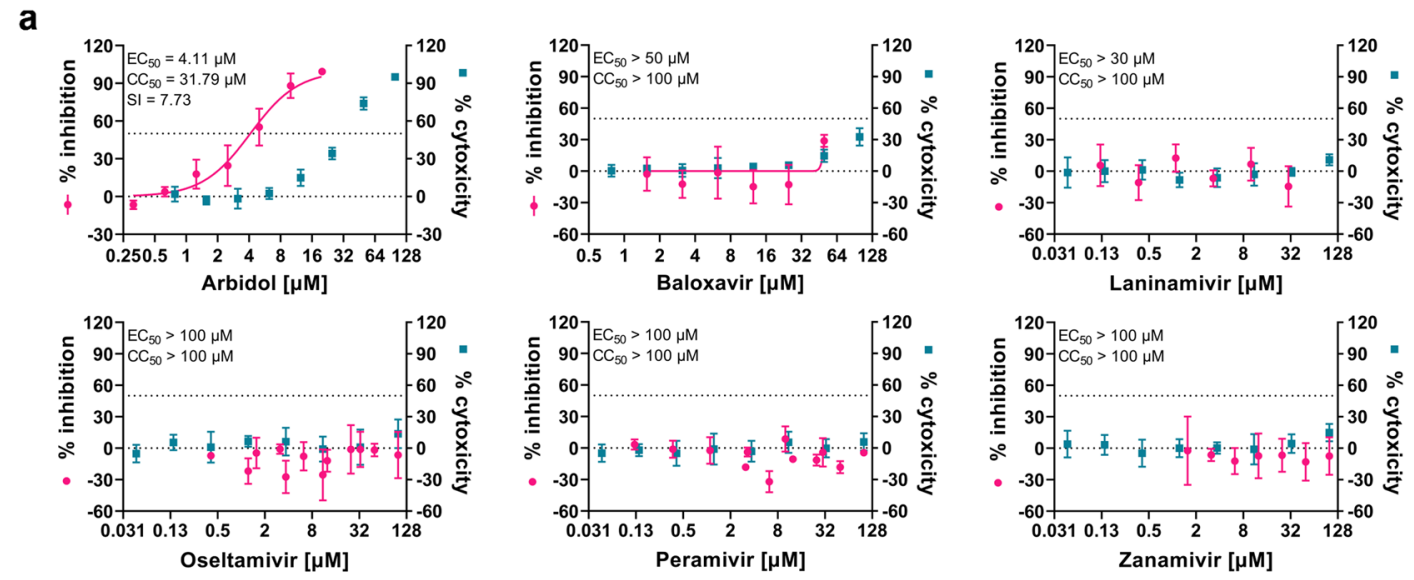

b

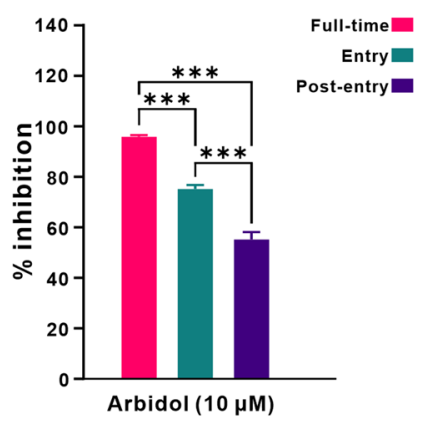

C
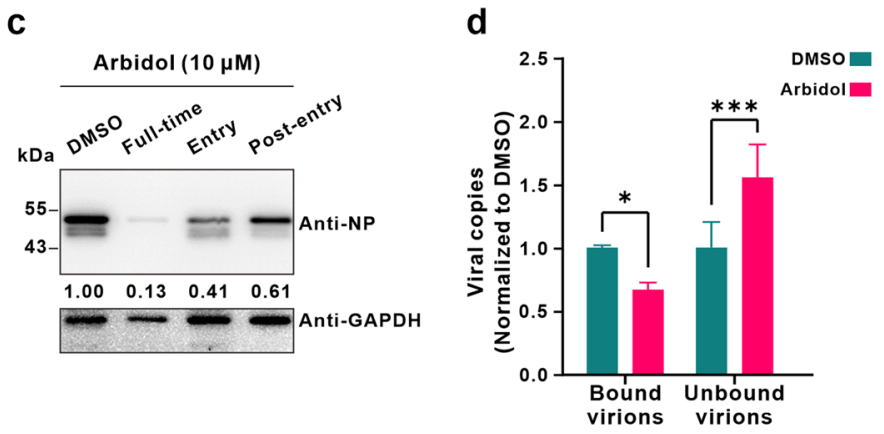

e

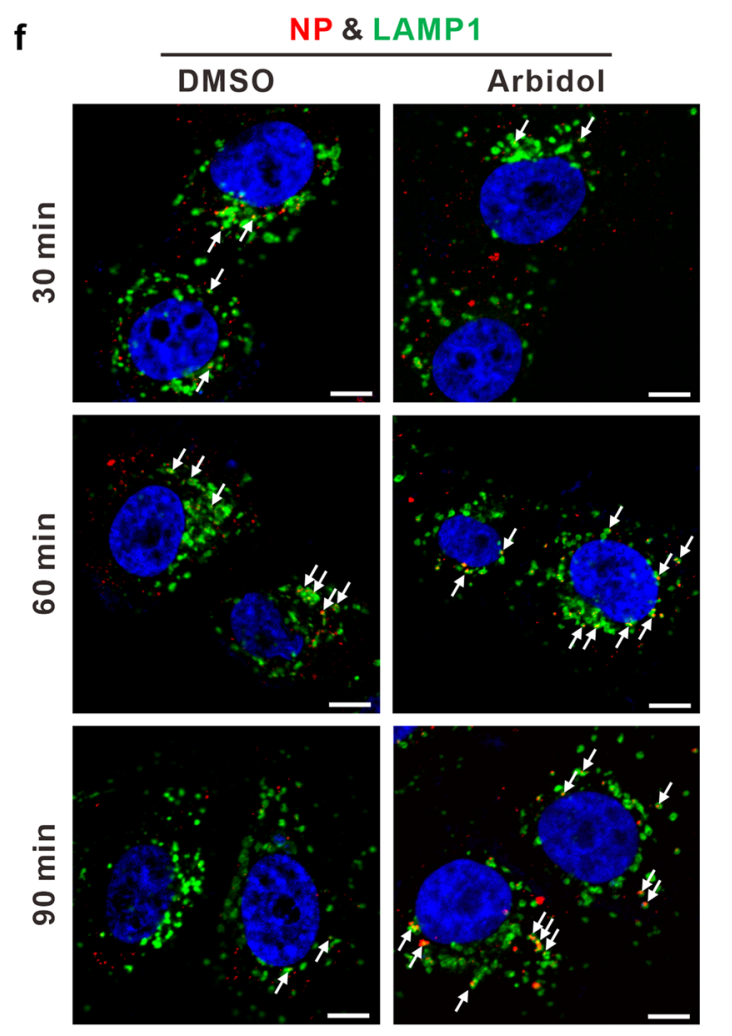

Fig. 1 (See legend on next page.) 
(see figure on previous page)

Fig. 1 Comparative antiviral efficacy of anti-influenza drugs and the mode of actions of arbidol against SARS-CoV-2 infection in vitro. a Antiviral activities of the drugs. The antiviral efficacy was evaluated in Vero E6 cells by qRT-PCR analysis of virus yield at $48 \mathrm{~h}$ p.i. Data represent the mean \pm standard deviation (SD) from two independent repeats. b, c Time-of-addition experiment of arbidol. Three experimental groups (Full-time, Entry, and Post-entry) were set up as described in the Supplementary Methods. At $16 \mathrm{~h} \mathrm{p.i.,} \mathrm{virus} \mathrm{yield} \mathrm{in} \mathrm{the} \mathrm{cell} \mathrm{supernatant} \mathrm{was} \mathrm{quantified} \mathrm{by} \mathrm{qRT-}$ PCR (b), and the expression of NP in infected cells was analyzed by western blots (c). The values below the blot represent the relative band intensity (NP/GAPDH) normalized to that of the DMSO group. $\mathbf{d}$ Impact of arbidol on SARS-CoV-2 binding. Vero E6 cells were treated with arbidol (10 $\mu$ M) or DMSO for $1 \mathrm{~h}$ prior to infection with SARS-CoV-2 at $4^{\circ} \mathrm{C}$ for $1 \mathrm{~h}$. The supernatant (unbound virions) and the cells containing bound virions (bound virions) were collected for quantification of viral RNA copies by qRT-PCR. e, $\mathbf{f}$ Effect of arbidol on intracellular trafficking of SARS-CoV-2. The colocalization of virions with EEs or LEs was analyzed by immunofluorescence assays as described in the Supplementary Methods. e The portion of virions that co-localized with EEs or ELs in each group ( $n>150$ cells) was quantified by Image J. f Representative confocal microscopic images of virions (red) and LAMP1 ${ }^{+}$ELs (green) in each group. The nuclei (blue) were stained with Hoechst 33258 dye. White arrows: virions co-localized with ELs; bars: $10 \mu \mathrm{m}$. For (b) and (e), statistical analysis was performed using a one-way analysis of variance (ANOVA) with GraphPad Prism. For (d), statistical analysis was performed and calculated by unpaired two-tailed $t$ test. ${ }^{*} P<0.05$; ${ }^{* * *} P<0.001$; ns, not significant.

(CPE) and immunofluorescence staining of infected cells. As shown in Supplementary Fig. S1, at 48 h p.i. only in cells treated with arbidol, but not with the other five drugs, viral NP expression and CPE due to SARS-CoV-2 was substantially reduced. To be noted, we also tried some human lung cell lines, for example human embryo lung fibroblasts MRC-5 and lung cancer cell line Calu-3, however, they were not very efficient for SARS-CoV-2 replication, and therefore were not used for this study.

Apart from influenza virus, arbidol was reported to inhibit a wide array of viruses by interfering with multiple steps of the virus replication cycle ${ }^{7}$. The stage of SARS-CoV-2 replication targeted by arbidol was explored by conducting a preliminary time-of-addition experiment using virus at an MOI of 0.05 . Arbidol was incubated with cells during the virus entry process (Entry), the post-entry stages (Post-entry), or the entire process of infection (Full-time) and progeny virus yield was quantified by qRT-PCR. The data revealed that arbidol efficiently blocked both viral entry and post-entry stages. It had a profound impact on virus Entry $(\sim 75 \%$ inhibition) with a lesser effect on Post-entry events ( $55 \%$ inhibition rate) (Fig. 1b). In addition, western blot analysis (Fig. 1c) and immunofluorescence microscopy (Supplementary Fig. S2) confirmed that the expression level of viral NP was reduced drastically at Full-time (13\% of the DMSO group, Fig. 1c), and showed more inhibitory effect at the Entry stage (41\%) than at the Post-entry stage (61\%).

The details of how arbidol blocks the entry of SARS$\mathrm{CoV}-2$ into cells were further investigated. Virus (MOI = 0.05 ) was allowed to bind to Vero E6 cells at $4{ }^{\circ} \mathrm{C}$ for $1 \mathrm{~h}$ in the presence of arbidol $(10 \mu \mathrm{M})$ or DMSO control. Virus particles bound to the cell (bound virions) and those in the supernatant (unbound virions) were analyzed by qRTPCR. The results showed that arbidol treatment led to a significantly decreased binding efficiency (67\%) compared with the control group $(P<0.05)$ (Fig. 1d). Correspondingly, the portion of unbound virions increased significantly to $156 \%$ of the control group after arbidol treatment $(P<0.001)$ (Fig. 1d).

Next, we analyzed viral intracellular trafficking. As we reported recently, within infected cells, SARS-CoV-2 underwent vesicle transportation, which was first carried out by early endosomes (EEs) then further transported to endolysosomes $(E L s)^{8}$. Co-localization of virions with EEs or ELs was visualized by immunofluorescence microscopy and statistically analyzed ( $n>150$ cells). As shown in Fig. 1e and Supplementary Fig. S3, in each tracked time points, there was no significant difference in the amounts of virions co-localized with EEs when comparing the DMSO- and arbidol-treated groups, although as time of infection went on $(30,60$, and 90 min p.i.), the levels of colocalization considerably decreased in both DMSO(24.0\%, 5.1\%, and 3.2\%) and arbidol- (21.4\%, 4.1\%, and $2.8 \%$ ) treated groups, suggesting that some virions were already transported from EEs to the next stage of vesicle transportation. By contrast, at $60 \mathrm{~min}$ p.i., a slightly higher percentage of virions were transported to ELs in the arbidol-treated group (22.4\%) than in the DMSO group $(18.3 \%)(P<0.05)$ (Fig. 1e, f). At 90 min p.i., significantly fewer virions ( $13.5 \%)$ were detected in ELs in the DMSO group; whereas significantly higher proportions of virions $(\sim 23.6 \%)$ remained within ELs in the arbidol-treated group, suggesting the drug trapped the virus in the ELs $(P<0.001)$ (Fig. 1e, f). Taken together, these results suggested that arbidol impeded not only viral attachment, but also release of SARS-CoV-2 from intracellular vesicles (ELs).

Among the drugs tested, laninamivir, oseltamivir, peramivir, and zanamivir are neuraminidase (NA) inhibitors, which are most widely prescribed for prophylaxis and treatment of influenza. Although no NA analog exists in SARS-CoV-2, NA inhibitors such as oseltamivir nevertheless are being used clinically in treating COVID-19 patients $^{1,2}$. Our data showed these NA inhibitors were not active against SARS-CoV-2 (Fig. 1a), which is consistent with the finding that oseltamivir and zanamivir were 
ineffective in inhibiting SARS-CoV ${ }^{9}$. Baloxavir marboxil is a new anti-influenza drug, which selectively inhibits the endonuclease activity of the viral polymerase responsible for snatching capped primers from host mRNAs to initiate viral mRNA transcription. However, this "capsnatching" mechanism of the endonuclease is not shared by coronaviruses that encode their own enzymes to form $5^{\prime}$-mRNA cap structures ${ }^{10}$. This may explain why baloxavir failed to block SARS-CoV-2 infection (Fig. 1a). During the review process of this study, Choy et al. also showed that oseltamivir and baloxavir failed to inhibit SARS-CoV-2 in vitro ${ }^{11}$.

Arbidol, an indole-derivative, has been licensed for decades in Russia and China against influenza. It is a broad-spectrum drug against a wide range of enveloped and non-enveloped viruses. Arbidol interacts preferentially with aromatic amino acids, and it affects multiple stages of the virus life cycle, either by direct targeting viral proteins or virus-associated host factors ${ }^{7}$. For example, in influenza virus, crystal structures showed that arbidol inserted into a hydrophobic pocket of the fusion subunit of HA, thus hindering low-pH conformational change of $\mathrm{HA}$ and blocking the fusion process ${ }^{12}$. In hepatitis $\mathrm{C}$ virus, arbidol impaired both virus attachment and intracellular vesicle trafficking ${ }^{13}$. Likewise, we found arbidol plays a role in interfering SAS-CoV-2 binding (Fig. 1d) and intracellular vesicle trafficking (Fig. 1e, f). Arbidol can also bind to lipid membranes and may alter membrane configuration of the cytoplasm or the endosome, which are crucial for viral attachment and fusion ${ }^{7}$. It could be further investigated whether arbidol targets virus or/ and cells by using published method ${ }^{14}$.

In summary, among the six anti-influenza drugs, only arbidol efficiently inhibited SARS-CoV-2 infection. Functionally, it appears to block virus entry by impeding viral attachment and release from the ELs. Although the SI of arbidol is relatively low ( $\mathrm{SI}=7.73)$, as a repurposed drug, its pharmacokinetics profile such as maximal concentration $(\mathrm{Cmax})$ is more important for predicting efficacy. It is generally believed that if the Cmax achieves $\mathrm{EC}_{90}$, the drug is very likely to be effective; while if the Cmax achieves $\mathrm{EC}_{50}$, the drug is possibly effective in vivo. In humans, a single oral administration of $800 \mathrm{mg}$ of arbidol results in Cmax of $\sim 4.1 \mu \mathrm{M}^{15}$, and this dosage is efficacious and safe against different influenza viruses with $\mathrm{EC}_{50}$ values ranging from $2.5-20 \mu \mathrm{M}^{7,16}$. Arbidol also showed anti-inflammatory activity, which may enhance its efficacy in vivo ${ }^{16}$. Considering the $\mathrm{EC}_{50}(4.11 \mu \mathrm{M})$ of arbidol against SARS-CoV-2 is comparable to, or even lower than those of influenza viruses, we, therefore, suggest that arbidol is potentially effective to treat COVID-19 patients. However, the current dose of arbidol $(200 \mathrm{mg}, 3$ times/day) recommended by the Chinese Guidelines may not be able to achieve an ideal therapeutic efficacy to inhibit SARS-CoV-2 infection, and should be elevated. This needs to be verified by clinical trials.

\section{Acknowledgements}

We thank Prof. Zhenhua Zheng from Wuhan Institute of Virology for kindly providing the anti-LAMP1 rabbit polyclonal antibody; Beijing Savant

Biotechnology Co., Itd for kindly providing the anti-NP monoclonal antibody; Jia Wu, Jun Liu, and Hao Tang from BSL-3 Laboratory, and Dr. Ding Gao from the Core Faculty of Wuhan Institute of Virology for their critical support; Dr. Basil Arif for scientific editing of the paper. This work was supported by grants from the National Science and Technology Major Projects for "Major New Drugs Innovation and Development" (2018ZX0971 1003), the National Key R\&D program of China (2020YFC0841700), and the National Natural Science Foundation of China (31621061).

\section{Author details}

${ }^{1}$ State Key Laboratory of Virology, Wuhan Institute of Virology, Center for Biosafety Mega-Science, Chinese Academy of Sciences, Wuhan 430071, China. ${ }^{2}$ National Engineering Research Center for the Emergency Drug, Beijing Institute of Pharmacology and Toxicology, Beijing 100850, China. ${ }^{3}$ University of the Chinese Academy of Sciences, Beijing 100049, China. ${ }^{4}$ National Virus Resource Center, Wuhan Institute of Virology, Chinese Academy of Sciences, Wuhan 430071, China

\section{Author contributions}

M.W., W.Z., and Z.H. conceived and designed the experiments. X.W., R.C., H.Z., J. L., M.X., H.H., Y.L., L.Z., W.L., X.Y., Z.S., and F.D. participated in multiple experiments; M.W., Z.H., W.Z., X.W., R.C., H.Z., J.L., M.X., H.H. Y.L., and X.S. analyzed the data. M.W., R.C., and Z.H. wrote the paper. Z.H., M.W., and W.Z. provided the final approval of the paper.

\section{Conflict of interest}

The authors declare that they have no conflict of interest.

\section{Publisher's note}

Springer Nature remains neutral with regard to jurisdictional claims in published maps and institutional affiliations.

Supplementary Information accompanies the paper at (https://doi.org/ 10.1038/s41421-020-0169-8).

Received: 29 March 2020 Accepted: 11 April 2020

Published online: 02 May 2020

\section{References}

1. Chen, N. et al. Epidemiological and clinical characteristics of 99 cases of 2019 novel coronavirus pneumonia in Wuhan, China: a descriptive study. Lancet 395, 507-513 (2020).

2. Huang, C. et al. Clinical features of patients infected with 2019 novel coronavirus in Wuhan, China. Lancet 395, 497-506 (2020).

3. Wang, M. et al. Remdesivir and chloroquine effectively inhibit the recently emerged novel coronavirus (2019-nCoV) in vitro. Cell Res. 30, 269-271 (2020).

4. Wang, Z., Yang, B., Li, Q., Wen, L. \& Zhang, R. Clinical Features of 69 Cases with Coronavirus Disease 2019 in Wuhan, China. Clin. Infect. Dis. ciaa272 (2020).

5. De Clercq, E. \& Li, G. Approved antiviral drugs over the past 50 years. Clin. Microbiol. Rev. 29, 695-747 (2016).

6. Gasparini, R., Amicizia, D., Lai, P. L., Bragazzi, N. L. \& Panatto, D. Compounds with anti-influenza activity: present and future of strategies for the optimal treatment and management of influenza. Part I: Influenza life-cycle and currently available drugs. J. Prev. Med. Hyg. 55, 69-85 (2014).

7. Blaising, J., Polyak, S. J. \& Pecheur, E. I. Arbidol as a broad-spectrum antiviral: an update. Antivir. Res. 107, 84-94 (2014).

8. Liu, J. et al. Hydroxychloroquine, a less toxic derivative of chloroquine, is effective in inhibiting SARS-CoV-2 infection in vitro. Cell Discov. 6, 16 (2020).

9. Tan, E. L. et al. Inhibition of SARS coronavirus infection in vitro with clinically approved antiviral drugs. Emerg. Infect. Dis. 10, 581-586 (2004). 
10. Decroly, E., Ferron, F., Lescar, J. \& Canard, B. Conventional and unconventional mechanisms for capping viral mRNA. Nat. Rev. Microbiol. 10, 51-65 (2011).

11. Choy, K. T. et al. Remdesivir, lopinavir, emetine, and homoharringtonine inhibit SARS-CoV-2 replication in vitro. Antiviral Res. 178, 104786 (2020).

12. Kadam, R. U. \& Wilson, I. A. Structural basis of influenza virus fusion inhibition by the antiviral drug Arbidol. Proc. Natl Acad. Sci. USA 114, 206-214 (2017).

13. Blaising, J. et al. Arbidol inhibits viral entry by interfering with clathrindependent trafficking. Antivir. Res. 100, 215-219 (2013).
14. $\mathrm{Si}$, L. et al. Triterpenoids manipulate a broad range of virus-host fusion via wrapping the HR2 domain prevalent in viral envelopes. Sci. Adv. 4, eaau8408 (2018).

15. Sun, $Y$. et al. Pharmacokinetics of single and multiple oral doses of arbidol in healthy Chinese volunteers. Int. J. Clin. Pharmacol. Ther. 51, 423-432 (2013).

16. Liu, Q. et al. Antiviral and anti-inflammatory activity of arbidol hydrochloride in influenza A (H1N1) virus infection. Acta Pharmacol. Sin. 34, 1075-1083 (2013). 